\title{
Inhaltsverzeichnifs des neun und dreifsigsten Bandes, nach den Gegenständen.
}

\section{Reine Mathe in at ik.}

Nr. der Abhandluag.

3. Note sur un système de certaines formules. M. A. Cayley à Cambridge. I. 14

4. Note sur quelques formules qui se rapportent à la multiplication des fonctions elliptiques. Par le mème. . . . . . . . . . . . . . . . . .

5. Über die Behandlung der Lebre der reellen Factoriellen und Facultāten nach einer Methode der Einschliefsung in Grenzen. Von Herrn Dr. M. Ohm, Prof. der Mathematik an der Universităt zu Berlin.

7. De integralibus

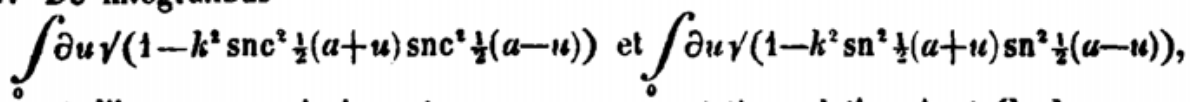
et aliis, quae cum ipsis sunt connexa, commentatio analytica. Auct. Gudermann, Monast. Guelph.

I. 50

8. Ableitung einiger bestimmten Integrale aus den Formeln der Abhandlung No. 18. im 37ten Bande dieses Journals. Von Herrn Dr. Dienger in Sinsheim bei Heidelberg. . . . . . . . . . . . . . . . . . .

9. Einiges zur Zahlenlehre. Von demselben. . . . . . . . . . . . I. 67

12. Moyens pour trouver l'expression de la $n^{\text {iène }}$ intégrale particulière de l'équation linéaire

$$
\boldsymbol{y}^{(n)}+\mathbf{P y}^{(n-1)}+Q \boldsymbol{Q}^{(n-2)}+\ldots .+S_{y^{\prime}}+T_{y}=0,
$$

à l'aide des $n-1$ valeurs $y_{1}, y_{2}, \ldots y_{n-1}$ qui satisfont à cette équation. Par Mr. le Dr. C. J. Malmstèn, prof. des Mathém. à l'Univers. d’Upsảla.

13. De l'équation différentielle

$$
x^{n-1}\left(a_{n}+b_{n} x\right) \cdot y^{(n)}+x^{n-2}\left(a_{n-1}+b_{n-1} x\right) \cdot y^{(n-1)}+\cdots+\left(a_{1}+b_{1} x\right) y^{\prime}+b_{0} y=0 \text {. }
$$

Par le même.

14. De l'équation différentielle

$$
y_{x}^{\prime \prime}+\frac{y_{x}^{\prime}}{x}+\lambda s^{m} y=0
$$

Par le mème. . . . . . . . . . . . . . . . . . . . . . Il. 108

15. Note sur les fonctions Elliptiques. Par le mème. . . . . . . . . II. 116

16. Abrifs einer Theorie der elliptischen Functionen. Von Herrn Prof. E. Heine zu Bonn.

18. Zur Theorie der homogenen Functivien dritten Grades von drei Variabeln. Von Herrn S. Aronhold zu Berlin. . . . . . . . . . . . . .

19. Ober die Irreductibilitāt und einige andere Eigenschaften der Gleichung, von welcher die Theilung der ganzen Lemniscate abhangt. Von Herrn Dr. G. Eisenstein zu Berlin.

20. Lehrsätze. Von demselben. . . . . . . . . . . . . . . . II. 180

22. Öber einige allgemeine Eigenschaften der Gleichung, von welcher die Theilung der ganzen Lemniscate abhangt, nebst Anwendungen derselben auf die Zahlentheorie. (Als Fortselzung der Abhandlung No. 19. in diesem Bande.) Von Herrn Dr. G. Eisenstein zu Berlin. . . . . . . . . III. 224

23. Schlurs dieser Abhandlung. . . . . . . . . . . . . IV. 275 
Nr. der

24. Über die in der Gau/sischen „Summatio quarumdam serierum singularium" vorkommenden Reihen. Von Herrm Prof. E. Heine in Bonn. . . . . IV. 288

25. Über die Reduction der quadratischen Formen auf die kleinste Anzahl Glieder. Von Herrn Prof. C. G. J. Jacobi zu Berlin. (Gelesen in der Berliner Akademie der Wissenschaften am 9ten November 1848.) . . . . . . .

27. Uber ein einfaches Mittel zur Auffindung der höheren Reciprocitătsgesełze und der mit ihnen zu verbindenden Ergänzungssãtze. Von Herrn Dr. G. Eisenstein, Docent an der Universilät zu Berlin.

2. G e o m e t r i e.

1. Note sur quelques formules relatives aux coniques. Par M. A. Cayley à Cambridge. . . . . . . . . . . . . . . . . . . . . .

2. Sur le problème des contacts. Par le même. . . . . . . . . . .

6. Die Gesetze der Succession einer Reihe sphärischer Kreise, von welchen jeder den nächstfolgenden und zugleich zwei feste Kreise berūhrt, deren einer im Innern des andern enthalten ist. Von dem Herrn Professor Dr. Gudermanı zu Münster.

7. Thèorème relatif au cercle qui passe par trois points d'une ellipse. Par Mr. Joachimsthal de Berlin. . . . . . . . . . . . . . . . .

19. Ober die Irreductibilität und einige andere Eigenschaften der Gleichung, von welcher die Theilung der ganzen Lemniscate abhangt. Von Herrn Dr. G. Eüsenstein zu Berlin.

22. Über einige allgemeine Eigenschaften der Gleichung, von welcher die Theilung der ganzen Lemniscate abhangt, nebst Anwendung derselben auf die Zahlentheorie. (Als Fortselzung der Abhandlung No. 19. in diesem Bande.) Von demselben. . . . . . . . . . . . . . . . . . IIl. 224

23. Schlufs dieser Abhandlung. . . . . . . . . . . . . . . . . . . . IV. 275

28. Lettre de Mr. G. Salmon de Dublin à l'éditeur de ce journal. . . . . IV. 365

$$
\text { 3. } \mathrm{M} \text { e c h a } \mathrm{n} \mathrm{i} \text {. }
$$

26. Sur la rotation d'un corps. Extrait d'une lettre addresséc à l'académie des sciences de Paris par Mr. C. G. J. Jacobi (lu dans la séance du 30 juillet 1849).

\section{Angew andte $M$ athematik.}

10. An Essay on the Application of mathematical Analysis to the theories of Electricity and Magnetism. By the late George Green, fellow of Gonvilleand Cains-Colleges at Cambridge. . . . . . . . . . . . . . . . 1.73

21. Über Sparcassen. Vom Heransgeber. . . . . . . . . . . . . . III. 183

\section{Verschiedenes.}

11. Notiz. Von Herrn Dr. Prcu/s, Königl. Professor und Historiographen zu Berlin.

Fac simile einer Handschrift von Klïgel. . . . . . . . . . . . . . . . . . 1 .

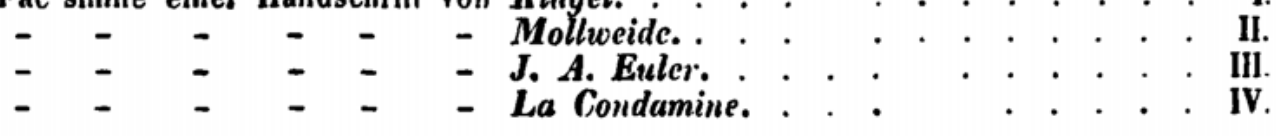

